\title{
Surfactant protein A induces the pathogenesis of renal fibrosis through binding to calreticulin
}

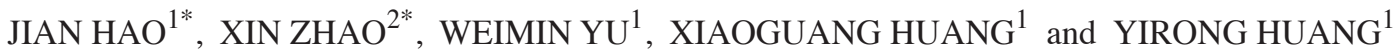 \\ ${ }^{1}$ Department of Nephrology, Shanxi Dayi Hospital, Taiyuan, Shanxi 030032; \\ ${ }^{2}$ Department of Radiotherapy, The Second Hospital of Shanxi Medical University, \\ Taiyuan, Shanxi 030001, P.R. China
}

Received April 19, 2017; Accepted October 25, 2017

DOI: $10.3892 /$ etm.2018.6919

\begin{abstract}
Renal fibrosis is a significant characteristic of chronic kidney diseases. Surfactant protein A (SP-A) is a recently identified fibrosis-associated factor in lung fibrosis; however, whether SP-A has the same role in renal fibrosis has remained elusive. The aim of the present study was to investigate the role of SP-A and its receptor calreticulin (CRT) in the pathogenesis of kidney fibrosis. The HK-2 human tubular epithelial cell line was cultured and treated with SP-A and SP-A + anti-CRT. The production of reactive oxygen species (ROS) at 30, 60 and $120 \mathrm{~min}$ was examined. Furthermore, cell apoptosis was assessed using an Annexin V assay and the expression of various proteins was measured using western blot analysis. In addition, the cell culture supernatants were collected and the expression of type I collagen was examined using ELISA. Compared with the control group, SP-A treatment significantly increased the ROS production, type I collagen secretion and cell apoptosis, which was partially inhibited by addition of anti-CRT. Furthermore, downregulation of matrix metalloproteinase (MMP)2 and -9 as well as upregulation of tissue inhibitor of metalloproteinase 1 indicated that SP-A treatment increased the degree of fibrosis in HK-2 cells, while addition of anti-CRT alleviated the fibrotic conditions. Finally, SP-A treatment significantly increased the expression of phosphorylated (p)-p38, p-p-65 and NADPH oxidase 2, which was partially inhibited by addition of anti-CRT. In conclusion, SP-A may participate in the pathogenesis of kidney fibrosis through binding to CRT and activate the mitogen-activated protein kinase/nuclear factor- $\kappa \mathrm{B}$-associated oxidative stress signaling pathway.
\end{abstract}

Correspondence to: Dr Jian Hao, Department of Nephrology, Shanxi Dayi Hospital, 99 Dragon Street, Taiyuan, Shanxi 030032, P.R. China

E-mail: haojian@mail.com

*Contributed equally

Key words: kidney fibrosis, SP-A, calreticulin, MAPK, NF-кB, oxidative stress

\section{Introduction}

Renal fibrosis is a common outcome of virtually all progressive kidney diseases. It includes renal interstitial fibrosis and glomerular sclerosis. Renal interstitial fibrosis is the major cause of renal dysfunction. It is characterized by excessive accumulation of extracellular matrix (ECM) in renal interstitium and overproliferation of fibroblasts. Due to the high solute transport activity of renal tubular epithelial cells, sufficient energy supply by mitochondria is extremely important to maintain normal renal function. Therefore, renal tubular epithelial cells are sensitive to oxidative stress, which makes oxidative stress a key factor of renal fibrosis. In the injured kidney, levels of reactive oxygen species (ROS) may increase dramatically and cause damage to the cell structure. Multiple signaling pathways are involved in ROS transduction. Nuclear factor $\kappa$-light-chain-enhancer of activated $\mathrm{B}$ cells $(\mathrm{NF}-\kappa \mathrm{B})$ is a known transcriptional factor that is redox-regulated by $\operatorname{ROS}$ (1). Activation of $N F-\kappa B$ triggers a series of cellular processes, including inflammation, immunity, cell proliferation and apoptosis.

Surfactant protein A (SP-A) is a newly identified factor that has been associated with pulmonary fibrosis $(2,3)$. SP-A is encoded by two homologous genes: SFTPA1 encodes SP-A1 and SFTPA 2 encodes SP-A2 (4). SP-A acts in a dual manner depending on the binding orientation. When SP-A binds signal regulatory protein $\alpha$, the $N F-\kappa B$ pathway is inhibited to prevent damage from excessive expression of inflammation factors; when SP-A binds calreticulin (CRT), the production of proinflammatory mediators is stimulated (5). CRT, also known as calregulin, CRP55 and calsequestrin-like protein, is a multifunctional protein that binds $\mathrm{Ca}^{2+}$ ions in endoplasmic and sarcoplasmic reticulum (6). Overexpression of CRT as a response to oxidative stress has been reported in previous studies (7-9).

A recent study reported that SP-A was also involved in renal fibrosis induced by unilateral ureter obstruction. SP-A expression was induced in kidney epithelium due to increased inflammation (10). Based on these previous results, the present study hypothesized that SP-A2 may contribute to the pathogenesis of renal fibrosis through binding to CRT and influence the oxidative stress response. 


\section{Materials and methods}

Cell culture and treatment. The human HK-2 cell line was obtained from the American Type Culture Collection (Manassas, VA, USA) and used as a model of proximal tubular cells. The cells were cultured in Dulbecco's modified Eagle's medium/F12 (Gibco; Thermo Fisher Scientific, Inc., Waltham, MA, USA) supplemented with $10 \%$ fetal bovine serum at $37^{\circ} \mathrm{C}$ in a humidified atmosphere containing $5 \% \mathrm{CO}_{2}$.

Cells were seeded into 6-well plates $\left(1.0 \times 10^{5}\right.$ cells/well) and allowed to attach by incubation for $24 \mathrm{~h}$. Subsequently, $10 \mu \mathrm{g} / \mathrm{ml} \mathrm{SP-A}$ was added, followed by incubation for 30 , 60 or $120 \mathrm{~min}$. SP-A2 was isolated from whole lung lavage fluid taken from patients with pulmonary alveolar proteinosis as previously described (11). The lung lavage fluid was used with the informed consent of 20 patients treated at Shanxi Dayi Hospital (Taiyuan, China) between January 2015 and October 2016. This study was approved by the ethics committee of Shanxi Dayi Hospital (Taiyuan, China). Cells without SP-A2 treatment were used as a negative control. To assess the role of SP-A in renal fibrosis through binding to CRT, the cells were also pre-treated with anti-CRT (10 $\mu \mathrm{g} /$ well; cat. no. sc-101436; 1:1,000; Santa Cruz Biotechnology Inc., Dallas, TX, USA) for $1 \mathrm{~h}$ and then treated with $10 \mu \mathrm{g} / \mathrm{ml}$ of SP-A2 for $1 \mathrm{~h}$. After the treatment, the cells were harvested for further assays.

Measurement of ROS production. HK-2 cells were incubated in 96-well plates $\left(1 \times 10^{4}\right.$ cells/well) and treated with SP-A2 with/without anti-CRT as described above. The production of ROS (mostly peroxide) was measured using a Reactive Oxygen Species Assay kit (Beyotime Institute of Biotechnology, Haimen, China). Fluorescence intensity was measured at an excitation wavelength of $488 \mathrm{~nm}$ and emission at $525 \mathrm{~nm}$ using a fluorescence microplate reader.

Measurement of type I collagen. The culture media were collected for detection of type I collagen. An ELISA kit for human type I collagen (Kamai Shu Biotechnology, China) was used according to the manufacturer's instructions.

Annexin $V$ assay. The apoptotic rate was detected using an Annexin V assay. Different cell groups were harvested with $0.25 \%$ trypsin and washed with PBS. After centrifugation for 5 min, cells were re-suspended and labeled using an Annexin V-FITC Apoptosis Detection kit (Beyotime Institute of Biotechnology) according to the manufacturer's instructions, followed by flow cytometric analysis.

Western blot analysis. The cells were treated and harvested as described above, then homogenized in radioimmunoprecipitation assay lysis buffer (Beyotime Institute of Biotechnology) and centrifuged at $12,000 \mathrm{x} \mathrm{g}$ at $4^{\circ} \mathrm{C}$ for $30 \mathrm{~min}$. The protein concentration was assayed via the bicinchoninic acid method. Protein samples (30 $\mu \mathrm{g}$ of each sample) were subjected to $10 \%$ SDS-PAGE and then transferred onto polyvinylidene difluoride membranes (EMD Millipore, Billerica, MA, USA), which were blocked with $5 \%$ skimmed milk at room temperature for $1 \mathrm{~h}$. The membranes were incubated overnight at $4^{\circ} \mathrm{C}$ with the following primary antibodies: Mouse monoclonal antibody to matrix
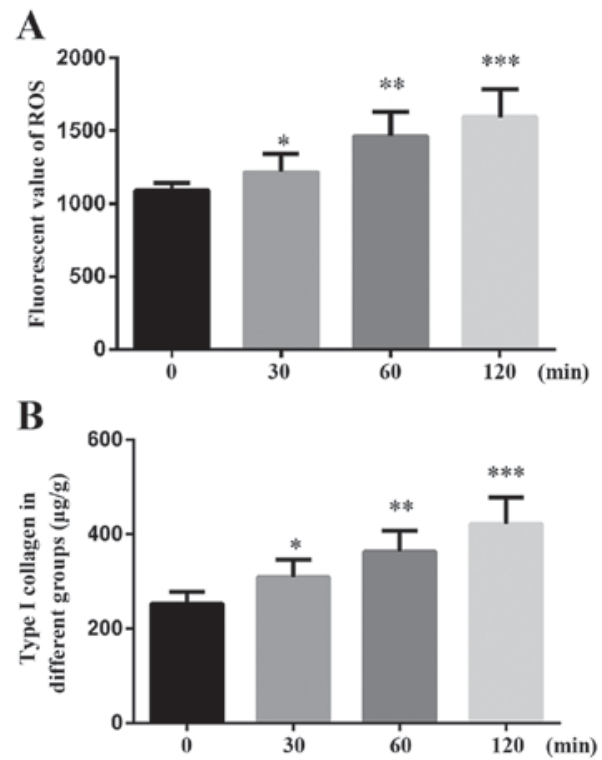

Figure 1. Production of ROS and the secretion of type I collagen in HK-2 cells induced by SP-A. HK-2 cells were incubated with $10 \mu \mathrm{g} / \mathrm{ml}$ SP-A for 30, 60 and $120 \mathrm{~min}$. SP-A induced (A) oxidative stress and (B) the secretion of type I collagen in a time-dependent manner. ${ }^{*} \mathrm{P}<0.05,{ }^{* *} \mathrm{P}<0.01,{ }^{* * *} \mathrm{P}<0.001$ vs. 0 min. ROS, reactive oxygen species; SP-A, surfactant protein A

metalloproteinase (MMP)2 (1:500 dilution; cat. no. sc-13594), MMP9 (1:500 dilution; cat. no. sc-21733), tissue inhibitor of matrix metalloproteinases (TIMP)-1 (1:500 dilution; cat. no. sc-21734), p38 mitogen-activated protein kinase (p38 MAPK; 1:500 dilution; cat. no. sc-136210), phosphorylated (p)-p38 MAPK (1:1,000 dilution; cat. no. sc-7973), p65 NF-кB (1:1,000 dilution; cat. no. sc-56735), p-p65 NF-кB (1:1,000 dilution; cat. no. sc-136548), NADPH oxidase 2 (NOX2; 1:500 dilution; cat. no. sc-130543) and GAPDH (1:1,000 dilution; cat. no. sc-47724). Subsequently, the membranes were incubated at room temperature for $1 \mathrm{~h}$ with horseradish peroxidase-conjugated secondary antibody to mouse immunoglobulin G (1:2,000 dilution; cat. no. sc-516102). All antibodies were purchased from Santa Cruz Biotechnology, Inc. Blots were detected using enhanced chemiluminescence (Beyotime Institute of Biotechnology, Haimen, China).

Statistical analysis. Each experiment was performed in triplicate and results were expressed as mean \pm standard deviation. Statistical analysis was performed using SPSS 20.0 software (IBM Corp., Armonk, NY, USA). The significance of differences between groups was determined using one-way analysis of variance followed by a post-hoc Dunnett's test. $\mathrm{P}<0.05$ was considered to indicate a statistically significant difference.

\section{Results}

SP-A induces oxidative stress and fibrosis in HK-2 cells. To determine whether SP-A induces oxidative stress in HK-2 cells, the effect of SP-A on ROS production was first examined. As presented in Fig. 1A, ROS production was increased in a time-dependent manner (30, 60 and $120 \mathrm{~min}$ ).

Furthermore, the amount of Type I collagen was also increased in a time-dependent manner, as demonstrated by the ELISA (Fig. 1B). 
A

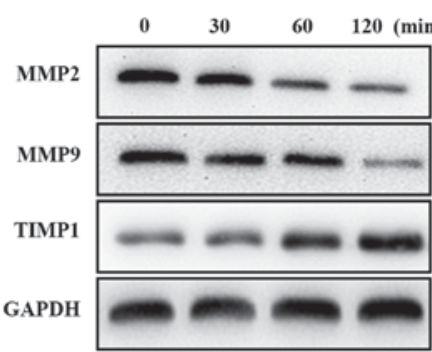

C

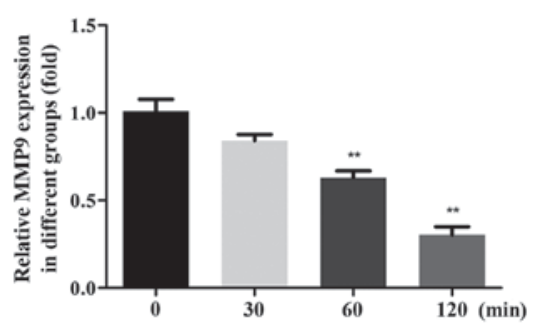

B

D
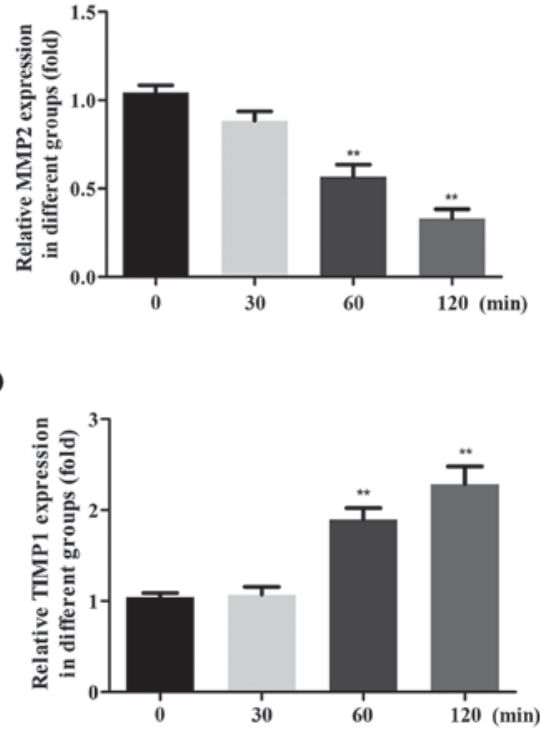

Figure 2. Expression of MMP2, MMP9 and TIMP1 protein. HK-2 cells were incubated with $10 \mu \mathrm{g} / \mathrm{ml}$ surfactant protein A for 30,60 or 120 min. (A) Representative western blot of MMP2, MMP9 and TIMP1. (B-D) Relative protein levels of (B) MMP2, (C) MMP9 and (D) TIMP1. GAPDH was used as an internal reference. ${ }^{* *} \mathrm{P}<0.01$ vs. 0 min. MMP, matrix metalloproteinase; TIMP1, tissue inhibitor of MMPs.

A

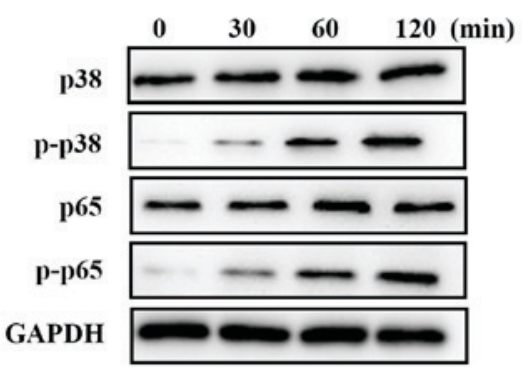

B

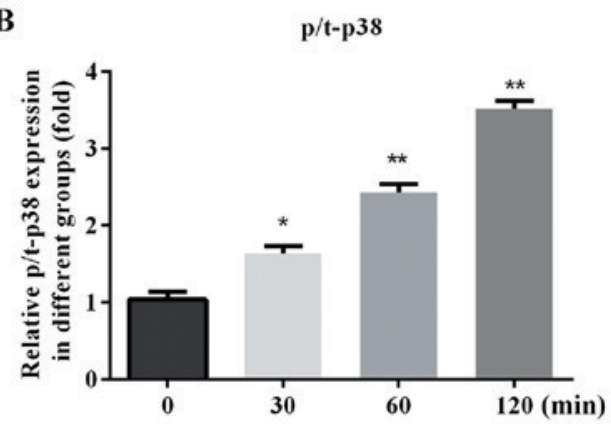

C

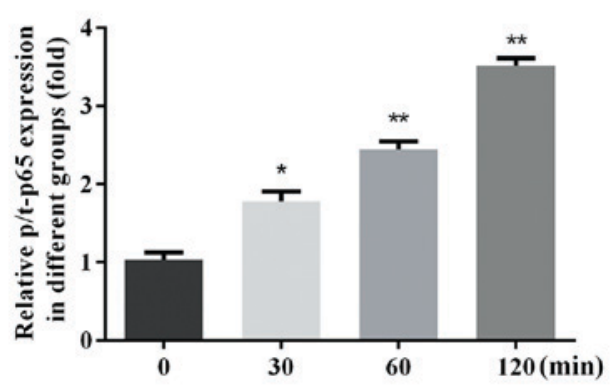

Figure 3. Levels of p38,p-p38, p65 and p-p65 protein. HK-2 cells were incubated with $10 \mu \mathrm{g} / \mathrm{ml}$ surfactant protein A for 30,60 or $120 \mathrm{~min}$. (A) Representative western blot bands for p38, p-p38, p65 and p-p65. Relative protein levels of (B) $\mathrm{p} / \mathrm{t}-\mathrm{p} 38$ and (C) p/t-p65. GAPDH was used as an internal reference. ${ }^{*} \mathrm{P}<0.05$ ${ }_{* * *} \mathrm{P}<0.01$ vs. 0 min. p-p38, phosphorylated p38; p-p65, phosphorylated p65.

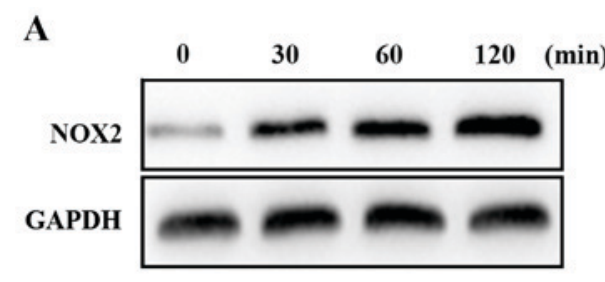

B

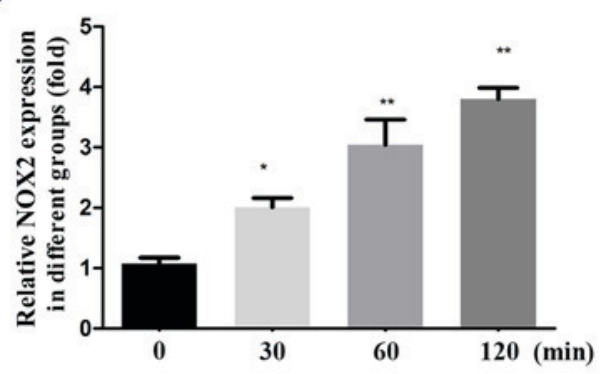

Figure 4. Expression of NOX2 protein. HK-2 cells were incubated with $10 \mu \mathrm{g} / \mathrm{ml}$ surfactant protein A for 30,60 or 120 min. (A) Representative western blot bands NOX2. (B) Relative protein levels of NOX2. GAPDH acted as an internal reference. ${ }^{*} \mathrm{P}<0.05,{ }^{* * *} \mathrm{P}<0.01$ vs. $0 \mathrm{~min}$. NOX2, NADPH oxidase 2 .

To characterize the effects of SP-A in inducing renal fibrosis by MMPs, western blot analysis was performed to measure the protein levels of MMP2, MMP9 and TIMP1. The expression of MMP2 and MMP9 protein was decreased, while that of TIMP1 protein was increased in a time-dependent manner (Fig. 2).

The mechanism of the renal fibrosis induction effect of SP-A via the MAPK/NF- $\kappa \mathrm{B}$ signaling pathways was further verified by assessing the levels of MAPK p38, MAPK p-p38, $\mathrm{NF}-\kappa \mathrm{B}$ p 65, NF- $\mathrm{B}$ p-p65 and NOX2 protein within total cell lysate. The expression of p/t-p38, p/t-p65 and NOX2 proteins was gradually increased by SP-A in a time-dependent manner (Figs. 3 and 4). 
A

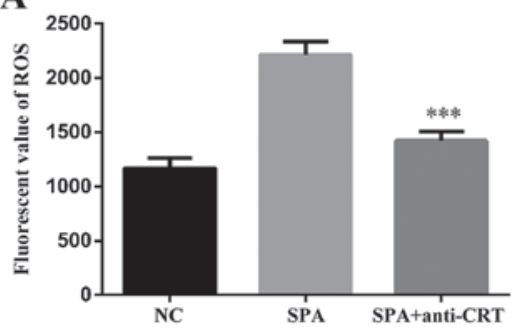

B

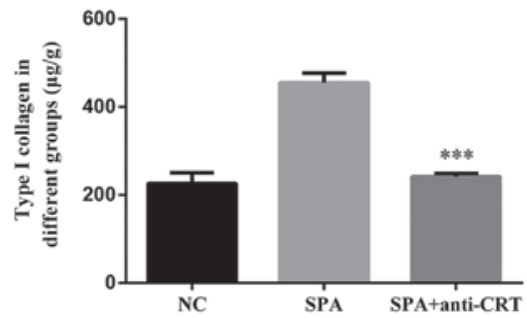

Figure 5. Effects of anti-CRT on the production of ROS and the secretion of type I collagen in SP-A-induced HK-2 cells. HK-2 cells were pre-treated with anti-CRT and then incubated with $10 \mu \mathrm{g} / \mathrm{ml} \mathrm{SP}$-A for $60 \mathrm{~min}$. (A) Anti-CRT treatment attenuated the production of ROS in a time-dependent manner (B) Anti-CRT treatment relieved the secretion of type I collagen in a time-dependent manner. ${ }^{* * *} \mathrm{P}<0.001$ vs. SP-A group. SP-A, surfactant protein A; ROS, reactive oxygen species; CRT, calreticulin; NC, normal control.

A

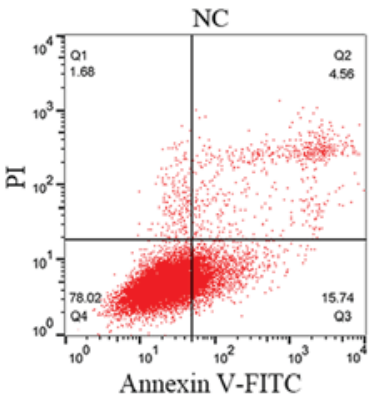

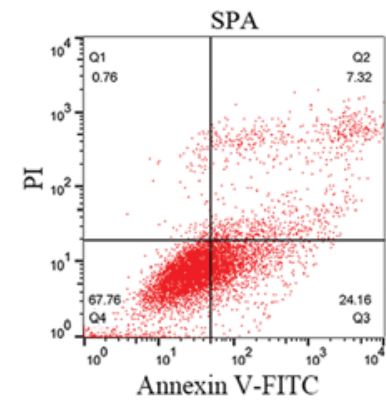

B

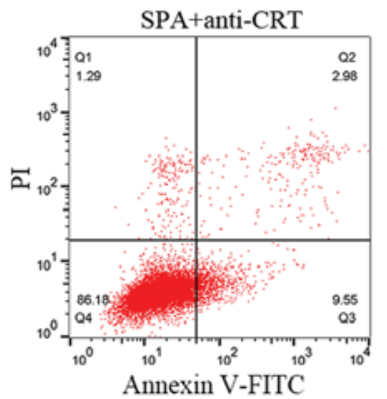

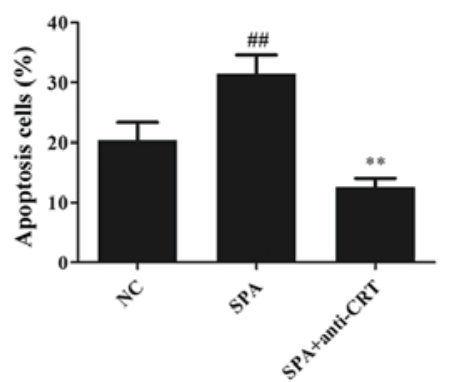

Figure 6. Cell apoptosis was measured using an Annexin V assay. (A) Flow cytometry results. (B) Apoptotic rate in different groups. ${ }^{\sharp \#} \mathrm{P}<0.01 \mathrm{vs.} \mathrm{NC}$; ${ }^{* *} \mathrm{P}<0.01$ vs. SP-A group. SP-A, surfactant protein A; NC, normal control; FITC, fluorescein isothiocyanate; PI, propidium iodide; CRT, calreticulin.

A

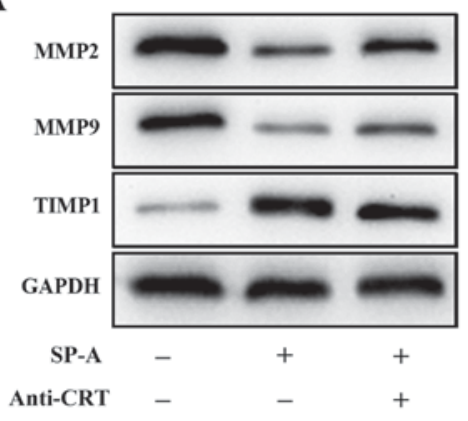

C

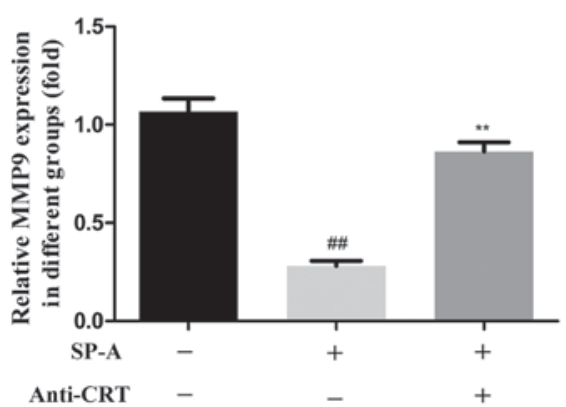

B

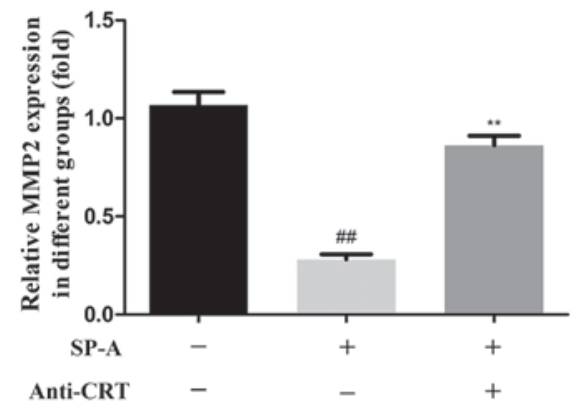

D

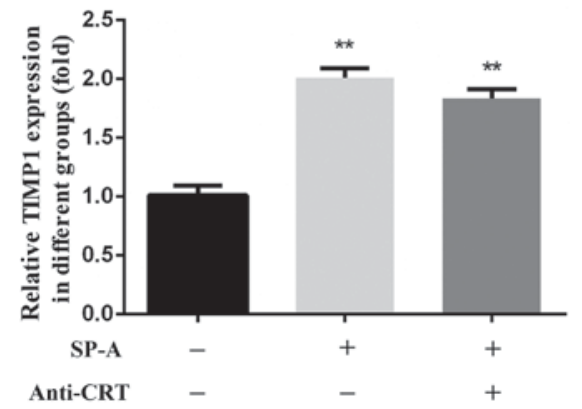

Figure 7. Effects of anti-CRT on the expression of MMP2, MMP9 and TIMP1 protein. HK-2 cells were pre-treated with anti-CRT and then incubated with $10 \mu \mathrm{g} / \mathrm{ml}$ SP-A for $60 \mathrm{~min}$. (A) Western blot bands for MMP2, MMP9 and TIMP1. Relative protein levels of (B) MMP2, (C) MMP9 and (D) TIMP1. GAPDH was used as an internal reference. ${ }^{\# \#} \mathrm{P}<0.01$ vs. $\mathrm{NC}$; ${ }^{* *} \mathrm{P}<0.01$ vs. SP-A group. SP-A, surfactant protein A; NC, normal control; MMP, matrix metalloproteinase; CRT, calreticulin; TIMP1, tissue inhibitor of metalloproteinase 1.

These results indicated that SP-A induces oxidative stress and renal fibrosis in HK-2 cells.
Anti-CRT alleviates oxidative stress and fibrosis in $\mathrm{HK}-2$ cells induced by SP-A. To determine whether SP-A induces 
A

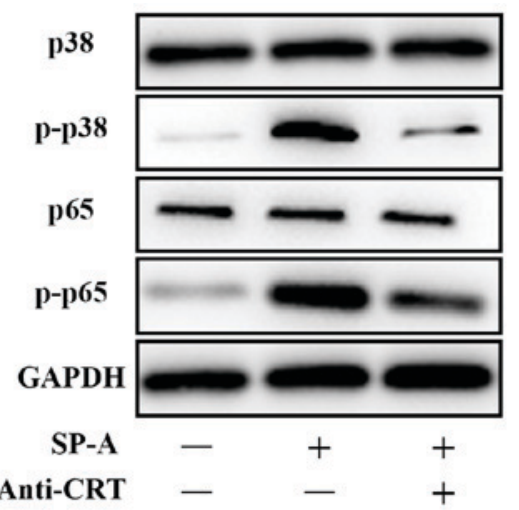

B

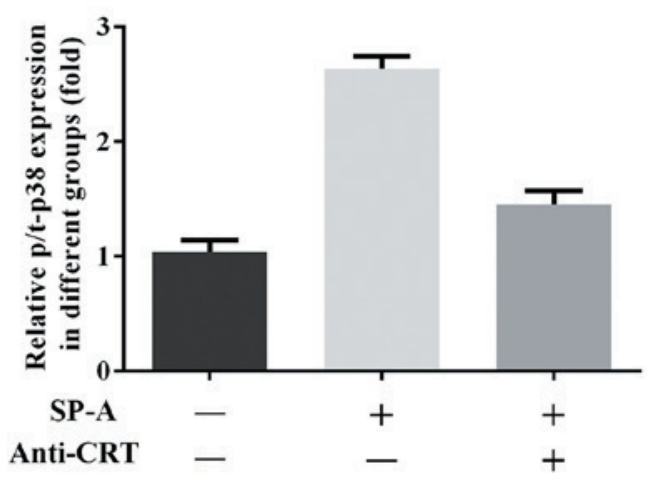

C

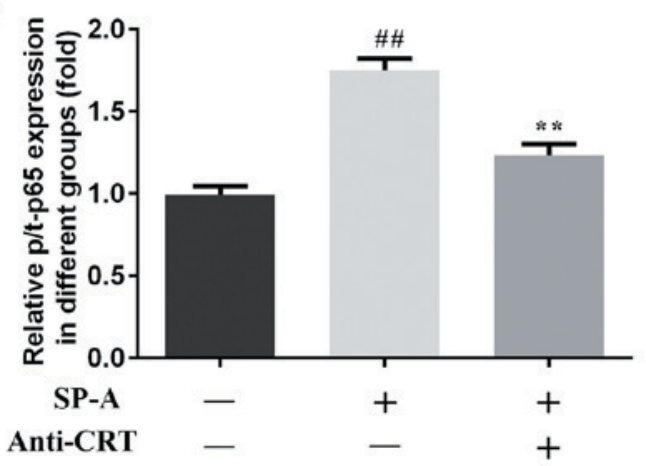

Figure 8. Effects of anti-CRT on the levels of p38, p-p38, p65 and p-p65 protein. (A) Representative western blot bands for $\mathrm{p} 38$, p-p38, p65 and p-p65. Relative protein levels of (B) p/t-p38 and (C) p/t-p65. GAPDH was used as an internal reference. ${ }^{\# \#} \mathrm{P}<0.01$ vs. NC; ${ }^{* *} \mathrm{P}<0.01$ vs. SP-A group. SP-A, surfactant protein A; CRT, calreticulin; NC, normal control; p-p38, phosphorylated p38; p-p65, phosphorylated p65.

oxidative stress through binding to CRT, HK-2 cells were pre-treated with anti-CRT for $1 \mathrm{~h}$, and the production of ROS and the content of type I collagen were assessed using a Reactive Oxygen Species Assay Kit and ELISA kit. Addition of anti-CRT obviously reduced the SP-A-induced production of ROS (Fig. 5A) and secretion of type I collagen by HK-2 cells (Fig. 5B). As presented in Fig. 6, compared with the control group, SP-A treatment significantly promoted cell apoptosis and anti-CRT partially alleviated this increase.

To characterize the effects of SP-A on MMPs through binding to CRT, the levels of MMP2, MMP9 and TIMP1 protein were assessed by western blot analysis. The addition of anti-CRT inhibited the SP-A-induced decreases in the protein expression of MMP2 and MMP9, and the increase in the expression of TIMP1 protein (Fig. 7).
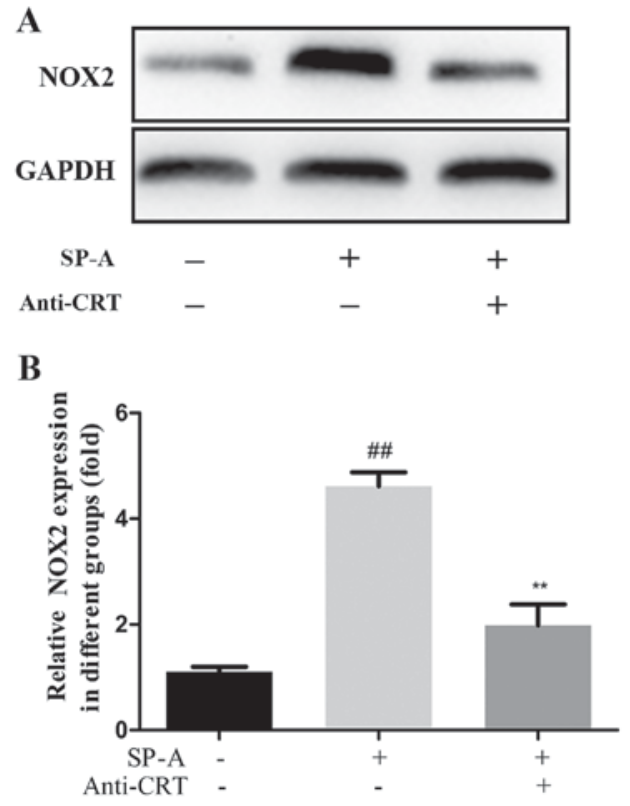

Figure 9. Effects of anti-CRT on the expression of NOX2 protein. (A) Representative western blot bands for NOX2. (B) Relative protein levels of NOX2. GAPDH was used as an internal reference. ${ }^{\# \#} \mathrm{P}<0.01$ vs. NC; ${ }^{* *} \mathrm{P}<0.01$ vs. SP-A group. SP-A, surfactant protein A; CRT, calreticulin; NC, normal control; NOX2, NADPH oxidase 2.

To further characterize whether SP-A has a significant role in renal fibrosis through binding to CRT, the levels of MAPK p38, MAPK p-p38, NF- $\kappa$ B p65, NF- $\kappa$ B p-p65 and NOX2 protein were assessed by western blot analysis. The expression of these proteins was increased by SP-A, and this increase was reversed by anti-CRT (Figs. 8 and 9).

These results suggested that anti-CRT alleviates oxidative stress and fibrosis in HK-2 cells induced by SP-A. Overall, SP-A has an important role in renal fibrosis through binding to CRT.

\section{Discussion}

The main features of chronic kidney disease are excessive deposition and fibrosis of ECM, leading to decreased renal function. Renal interstitial fibrosis is a common pathway and main pathological characteristic of chronic kidney disease progression to end-stage renal disease (12-14). SP-A is a novel factor that has been associated with pulmonary fibrosis $(2,3)$.

MMPs have a key role in the degradation of ECM and promote the degradation of ECM (particularly Type I and Type III collagen), while TIMPs have the effect of inhibiting the ECM, which reduces the degradation of the ECM and leads to fibrosis. MMPs/TIMPs have been confirmed to have a pivotal role in liver, myocardial and lung fibrosis (15-17). In the present study, western blot analysis was performed to determine the protein expression of MMP2, MMP9 and TIMP1. The results indicated that SP-A inhibits the expression of MMP2 and MMP9 and promotes the expression of TIMP1, while the addition of anti-CRT reduced these effects. In addition, ELISA indicated that SP-A promotes the secretion of Type I collagen, which was attenuated by the addition of anti-CRT. These results indicated that SP-A regulates renal fibrosis through binding to CRT. 
In the initial stage of renal fibrosis, numerous factors affect the redox state of renal cells and macrophages to stimulate the production of ROS. In the development stage of renal fibrosis, various stimuli lead to multiple fibrogenetic signaling. ROS are an important intracellular secondary messenger, activating the MAPK/NF- $\mathrm{B}$ signaling pathway $(1,18)$. Gardai et al $(5)$ demonstrated that SP-A regulates NF- $\mathrm{B}$ p65 and stimulates the production of proinflammatory mediators by binding to CRT. In the present study, SP-A treatment increased the production of ROS, and anti-CRT partially alleviated this increase. The western blot results illustrated that the protein

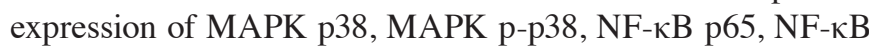
p-p65 and NOX2 was increased by SP-A, while this increase was inhibited by anti-CRT. These results confirmed that SP-A upregulates the expression of p65 and stimulates the production of proinflammatory mediators by binding to CRT.

In conclusion, the present results demonstrated that SP-A may participate in the pathogenesis of kidney fibrosis and activate the MAPK/NF- $\mathrm{B}$ oxidative stress signaling pathway by binding to CRT.

\section{Acknowledgements}

Not applicable.

\section{Funding}

No funding was received.

\section{Availability of data and materials}

The analyzed data sets generated during the present study are available from the corresponding author on reasonable request.

\section{Authors' contributions}

$\mathrm{XZ}$ wrote the manuscript and interpreted the data. JH, XZ and WY analyzed the data and revised the manuscript. $\mathrm{XH}$ and YH searched the literature and collected the data. JH designed the study. All authors read and approved the final manuscript.

\section{Ethics approval and consent to participate}

This study was approved by the Ethics Committee of Shanxi Dayi Hospital (Taiyuan, China).

\section{Patient consent for publication}

The lung lavage fluid was used with the informed consent of 20 patients treated at Shanxi Dayi Hospital (Taiyuan, China) between January 2015 and October 2016.

\section{Competing interests}

The authors declare that they have no competing interests.

\section{References}

1. Gloire G, Legrand-Poels S and Piette J: NF-kappaB activation by reactive oxygen species: Fifteen years later. Biochem Pharmacol 72: 1493-1505, 2006.

2. Samukawa T, Hamada T, Uto H, Yanagi M, Tsukuya G, Nosaki T, Maeda M, Hirano T, Tsubouchi $\mathrm{H}$ and Inoue H: The elevation of serum napsin A in idiopathic pulmonary fibrosis, compared with KL-6, surfactant protein-A and surfactant protein-D. BMC Pulm Med 12: 55, 2012.

3. Takahashi H, Fujishima T, Koba H, Murakami S, Kurokawa K, Shibuya Y, Shiratori M, Kuroki Y and Abe S: Serum surfactant proteins $\mathrm{A}$ and $\mathrm{D}$ as prognostic factors in idiopathic pulmonary fibrosis and their relationship to disease extent. Am J Respir Crit Care Med 162: 1109-1114, 2000.

4. Mccormack FX: Structure, processing and properties of surfactant protein A. Biochim Biophys Acta 1408: 109-131, 1998.

5. Gardai SJ, Xiao Y, Dickinson M, Nick JA, Voelker DR, Greene KE and Henson PM: By binding SIRPalpha or calreticulin/CD91, lung collectins act as dual function surveillance molecules to suppress or enhance inflammation. Cell 115: 13-23, 2003.

6. Zhang L, Wu G, Tate CG, Lookene A and Olivecrona G: Calreticulin promotes folding/dimerization of human lipoprotein lipase expressed in insect cells (Sf21). J Biol Chem 278: 29344-29351, 2003.

7. Zhang Y, Liu L, Jin L, Yi X, Dang E, Yang Y, Li C and Gao T: Oxidative stress-induced calreticulin expression and translocation: New insights into the destruction of melanocytes. J Invest Dermatol 134: 183-191, 2014.

8. Ihara Y, Urata Y, Goto S and Kondo T: Role of calreticulin in the sensitivity of myocardiac H9c2 cells to oxidative stress caused by hydrogen peroxide. Am J Physiol Cell Physiol 290: C208-C221, 2006.

9. Ihara Y, Kageyama K and Kondo T: Overexpression of calreticulin sensitizes SERCA2a to oxidative stress. Biochem Biophys Res Commun 329: 1343-1349, 2005.

10. Tian S, Li C, Ran R and Chen SY: Surfactant protein A deficiency exacerbates renal interstitial fibrosis following obstructive injury in mice. Biochim Biophys Acta 1863: 509-517, 2017.

11. Allen MJ, Voelker DR and Mason RJ: Interactions of surfactant proteins A and D with Saccharomyces cerevisiae and Aspergillus fumigatus. Infect Immun 69: 2037-2044, 2001.

12. Boor P, Ostendorf T and Floege J: Renal fibrosis: Novel insights into mechanisms and therapeutic targets. Nat Rev Nephrol 6: 643-656, 2010.

13. Farris $\mathrm{AB}$ and Colvin RB: Renal interstitial fibrosis: Mechanisms and evaluation. Curr Opin Nephrol Hypertens 21: 289-300, 2012.

14. Lawson J, Elliott J, Wheeler-Jones C, Syme H and Jepson R: Renal fibrosis in feline chronic kidney disease: Known mediators and mechanisms of injury. Vet J 203: 18-26, 2015.

15. Hemmann S, Graf J, Roderfeld M and Roeb E: Expression of MMPs and TIMPs in liver fibrosis-a systematic review with special emphasis on anti-fibrotic strategies. J Hepatol 46: 955-975, 2007.

16. Pei Z, Meng R, Li G, Yan G, Xu C, Zhuang Z, Ren J and Wu Z: Angiotensin-(1-7) ameliorates myocardial remodeling and interstitial fibrosis in spontaneous hypertension: Role of MMPs/TIMPs. Toxicol Lett 199: 173-181, 2010.

17. Manoury B, Nénan S, Guénon I, Lagente V and Boichot E: Influence of early neutrophil depletion on MMPs/TIMP-1 balance in bleomycin-induced lung fibrosis. Int Immunopharmacol 7: 900-911, 2007.

18. Jian KL, Zhang C, Shang ZC, Yang L and Kong LY: Eucalrobusone $\mathrm{C}$ suppresses cell proliferation and induces ROS-dependent mitochondrial apoptosis via the p38 MAPK pathway in hepatocellular carcinoma cells. Phytomedicine 25: 71-82, 2017. 